\title{
Conceptualización sobre el Desarrollo Sostenible: operacionalización del concepto para Colombia
}

\author{
Conceptualization on Sustainable Development: \\ Operationalization of the Concept for Colombia
}

Fecha de recepción: 12 de febrero de 2012 Fecha de evaluación: 22 de agosto de 2012 Fecha de aprobación: 27 de agosto de 2012

\author{
Mario Alejandro Pérez Rincón (Colombia) \\ Universidad del Valle \\ mario.perez@correounivalle.edu.co \\ Doctor en Ciencias Ambientales
}

\section{Resumen}

Este texto tiene como propósito desarrollar una discusión conceptual sobre el tema del desarrollo sostenible desde las perspectivas de la sostenibilidad débil y la sostenibilidad fuerte, que permita hacer recomendaciones para la operacionalización de este concepto en Colombia. El ensayo parte de una revisión histórica de las relaciones entre desarrollo y ambiente en el siglo XX; posteriormente se aborda el tema central del ensayo que corresponde a la discusión entre sostenibilidad débil y fuerte. Con base en este punto, se plantea una propuesta conceptual para operacionalizar este concepto en el país. Finalmente se entregan las conclusiones del debate en términos de recomendaciones de política.

\section{Palabras clave}

Capital intelectual, sociedad del conocimiento, aprendizaje, gestión del conocimiento, trabajadores, organizaciones inteligentes

\section{Abstract}

This article aims to develop a conceptual discussion on sustainable development from the perspective of weak and strong sustainability in order to make recommendations to operationalize the concept in Colombia. The essay starts from historical revision of the relations between development and environment in the XX century. Later, the main topic of the essay is approached: the discussion between weak and strong sustainability. Based on this, a conceptual proposal is stated to operationalize this concept in the country. Finally, conclusions are drawn from the debate in terms of policy recommendations.

\section{Keywords}

Sustainable development, ecosystems, environmental crisis, ecology, ethics. 


\section{Introducción}

A lo largo de su historia, el homo sapiens ha aprendido a aprovechar los ecosistemas del planeta y sus recursos para satisfacer sus necesidades materiales e inmateriales. Este éxito relativo como especie, en términos de conquistar el mundo, ha implicado un impacto muy fuerte sobre la biosfera y la geósfera, incrementando en forma creciente la presión antrópica sobre la oferta de bienes y servicios ambientales a medida que el ser humano intensifica su dominio sobre la naturaleza y se apropia de ella. Estos impactos se aceleraron a partir de la revolución industrial, acentuándose en el siglo pasado y en el presente, a tal punto que están agotando la capacidad del planeta de soportar los procesos socio-económicos, amenazando con ello la calidad de vida de la gente y la exitosa carrera del ser humano como especie dominante.

Las manifestaciones de la crisis ambiental se perciben en nuestra vida cotidiana en forma cada vez más clara, creciente y contundente. Los desórdenes del clima; el deshielo de los nevados; el desprendimiento de los grandes bloques de hielo de la Antártida; la contaminación de las aguas, el aire y el suelo; el agotamiento de los recursos naturales; la deforestación; la disminución de la biodiversidad; el inadecuado manejo y disposición de los residuos sólidos, entre otros; todos estos síntomas evidencian la crisis ambiental global y nacional. Pero además, ponen sobre el tapete dos realidades irrefutables: la existencia de límites asociados a la capacidad de soporte y asimilación de la naturaleza, aspecto que aún no ha podido integrarse en la mentalidad colectiva que todavía cree en la abundancia de recursos y servicios ambientales. Y la realidad de que somos parte de la naturaleza, y como tal debemos respetar unas reglas si queremos mantenernos como especie en el largo plazo.

La certeza sobre la existencia de límites en el mundo, manifestada a través de los problemas ambientales, es lo que ha permitido impulsar la sostenibilidad como el paradigma que intenta conciliar el conflicto entre la producción de bienes y servicios para la sociedad (desarrollo) y la oferta ambiental disponible en términos de recursos naturales y servicios ecosistémicos. La pregunta que surge entonces es: ¿cómo armonizar la búsqueda de mejor calidad de vida para el ser humano, con la necesidad de conservar la base ecosistémica y aceptar sus límites y reglas?, es decir, ¿cómo lograr una relación sostenible entre el mundo de las personas que pretende generar bienestar a toda la población y el mundo natural que soporta y posibilita la existencia de la sociedad y su actividad económica?

\section{Desarrollo y ambiente desde el siglo XX}

Han pasado más de 70 años desde que el concepto de 'desarrollo' se introdujo en el mundo académico y político cuando Schumpeter (1935) expuso el tema como objeto de trabajo en su libro El análisis del cambio económico, y luego el presidente Truman lo utilizó en un discurso ante las Naciones Unidas en 1949. Sin embargo, este concepto confluyó rápidamente hacia el crecimiento económico mediante una voluminosa producción de literatura (Domar, 1947; Ranis y Fei, 1952; Solow, 1956; Rostow, 1956; Barán y Hosbawn, 
1958), sin concebir en ningún momento la posibilidad de que el crecimiento pudiese tener límites'.

Al compás de estas teorías, el crecimiento económico adquirió un impulso inusitado abasteciendo de bienes y servicios a una población creciente, que incrementaba a la par sus niveles de consumo. Estos logros parciales, no solo generaron un alto grado de optimismo en la sociedad occidental, sino que facilitaron la difusión universal de los valores éticos del capitalismo: 1) la lógica individual de maximizar beneficios como propósito único de la vida, 2) la cultura del consumo y el dogma negligente, y 3) el arriesgado crecimiento económico perpetuo sin restricciones naturales, el cual se hace posible gracias a los avances del progreso tecnológico.

Con ello la economía adquiere su propia cosmogonía en el colectivo social en la mayor parte del mundo, generando

1 Existe una estrecha conexión entre modernización y desarrollo. Mientras el primero, ha sido quizás el referente principal para la sociología y los estudios culturales, el segundo hace referencia básicamente a la economía y la ciencia política. El desarrollo aparece como un programa de extensión universal de los beneficios del progreso científico y el bienestar material en el contexto internacional posterior a la Segunda Guerra Mundial, marcado por las necesidades de reconstrucción de Europa y Japón, por la descolonización y por la división del mundo en bloques. Aunque había estado desde mucho antes conectado con la confianza en el progreso, desde ese momento se asoció con una intervención consciente de ingeniería social y planificación encaminada a remodelar las sociedades a fin de acelerar su transición a la modernidad (García, 2004). El concepto de progreso por su parte, padre de todos estos conceptos, surge mucho antes en el siglo XVII asociado al triunfo del espíritu científico, traducido en la creencia de que la humanidad se había movido, se estaba moviendo y se movería siempre por la senda deseable de un mejoramiento material y espiritual indefinido (Naredo, 2003). una cultura económica específica: "la economía de mercado”. Como lo señala Escobar (1994):

la modernidad descansa no solo en una estructura epistemológica particular, sino en una serie de concepciones y prácticas llamadas económicas. La dinámica económica del siglo XIX y XX, basada en el individualismo y el utilitarismo, permitieron consolidar a la economía como una esfera real, autónoma, con sus propias leyes e independiente de lo político, lo social y lo cultural, y esto tiene consecuencias serias para la relación sociedad-naturaleza.

A la par de esta dinámica, aparecieron problemas a escala global y local. Los beneficios del crecimiento económico no se repartieron por igual entre países ni entre grupos sociales, ampliándose las brechas ${ }^{2}$. Pero además, la dinámica económica llevaba aparejada una demanda creciente de recursos naturales y procesos de contaminación que se fueron expandiendo, y difundiendo, especialmente los problemas ambientales a nivel global. La unificación creciente del mundo mostró que la globalización de los beneficios implicaba la globalización de los problemas (Sachs, 1996). El desarrollo y la acumulación debían prolongarse indefinidamente

2 La visión convencional del desarrollo ha sido cuestionada por las teorías de la dependencia que han puesto el acento al decir que los obstáculos al despegue no radican sólo en las resistencias tradicionales a la modernización (problemas estructurales internos) dentro de cada sociedad, sino también en una estructura económica internacional adversa, marcada por relaciones de dominio y de intercambio desigual entre dos bloques de países que coexisten en el sistema mundial: el centro y la periferia (Prebisch, 1949; Cardoso y Faletto, 1969; Wallerstein, 1974). 
en el tiempo, pero esta pretensión temporal chocó con la finitud del espacio del planeta Tierra. Entre más crecía la tasa de explotación, más rápido se hacían sentir los límites de la naturaleza a escala global.

Esta realidad fue reportada por diferentes estudios que llevaron a la sociedad y a las instituciones, a preocuparse por los crecientes problemas ambientales. Cinco obras pioneras en este campo, contribuyeron a generar un punto de inflexión histórica en relación con el cambio global: Silent spring (Carson, 1962); The economics of the coming spaceship earth (Boulding, 1966); The Population Bomb (Erlich, 1968); The entropy law and the economic process (Georgescu-Roegen N., 1971) y los Limites del crecimiento (Meadows et al, 1972). Estas obras de gran peso científico y ético, permitieron mostrar la capacidad de transformación del ambiente por parte de la especie humana, reviviendo la discusión malthusiana sobre el conflicto entre la dinámica del consumo y la dotación de recursos naturales $^{3}$. Igualmente, es necesario destacar a los movimientos ambientalistas norteamericanos que tenían como orientación el "culto a la vida silvestre", y que

3 En este escenario histórico, no puede desconocerse el papel jugado por la vocación sintética e integradora de la ecología (Haeckel, 1834-1919) y la termodinámica (Carnot, 1796-1832), visión que aunque data del siglo XIX, es en el siglo XX donde se desarrolla la ecología y la termodinámica de los sistemas abiertos a través de la "Teoría general de sistemas" que permitió extender estos enfoques sintetizadores a las más diversas disciplinas (Naredo, 2003). Ello posibilitó superar el análisis tradicional de aislar los elementos del universo para poder comprenderlos, y así cobró auge el argumento que para comprender no se requieren solo los elementos, sino las relaciones entre ellos. Nada puede aislarse convenientemente para ser estudiado por separado porque todo está interrelacionado (Bertalanffy, 1976). dieron origen posteriormente a organizaciones internacionales de defensa del medio ambiente como la Unión Internacional para la Conservación de la Naturaleza (IUCN, por sus siglas en inglés), el Worldwide Fund for Nature (WWF, por sus siglas en inglés), Nature Conservancy y Amigos de la Tierra.

La preocupación derivada de estos resultados a nivel mundial hizo que la ONU convocara la primera Conferencia Mundial sobre el Medio Ambiente en Estocolmo en 1972. A partir de allí, se han hecho diversos esfuerzos a nivel mundial por generar un alto nivel de conciencia y compromiso político sobre el problema ambiental y sus causas y consecuencias, reiterando todos la creciente intensidad del problema de deterioro ambiental y el papel que ha jugado en ello la actividad económica y los patrones de consumo (ver tabla 1).

Sin embargo, quizá el hito histórico más destacado desde el punto de vista que nos ocupa, fue la creación de la Comisión Mundial sobre Ambiente y Desarrollo (1983), que presentó su informe final en 1987, conocido como el Informe Brundtland, el cual se acuñó la expresión "Desarrollo Sostenible". Esta expresión fue definida como "el desarrollo que satisface las necesidades del presente sin comprometer la capacidad de las generaciones futuras de satisfacer sus propias necesidades" (WCED, 1987). Es evidente que la formulación del concepto de desarrollo sostenible ha conquistado un espacio socio-lingüístico sin precedentes; pocas expresiones, en un periodo tan corto desde su creación, han tenido tanto éxito (Boada y Toledo, 2003). El Informe Brundtland se ha convertido en referencia universal para la elaboración de estrategias y políticas de desarrollo eco-compatibles. 
Tabla 1. Eventos relevantes a nivel

mundial sobre medio ambiente

\begin{tabular}{|l|l|}
\hline \multicolumn{1}{|c|}{ Año } & \multicolumn{1}{c|}{ Evento } \\
\hline 1972 & $\begin{array}{l}\text { Conferencia Mundial sobre Medio Am- } \\
\text { biente (ONU) }\end{array}$ \\
\hline 1980 & $\begin{array}{l}\text { Estrategia Mundial para la Conserva- } \\
\text { ción (UICN y otros) }\end{array}$ \\
\hline 1983 & $\begin{array}{l}\text { Comisión Mundial sobre Ambiente y } \\
\text { Desarrollo (ONU) }\end{array}$ \\
\hline 1992 & $\begin{array}{l}\text { Cumbre mundial sobre Ambiente y De- } \\
\text { sarrollo de Río de Janeiro (ONU) }\end{array}$ \\
\hline 2002 & $\begin{array}{l}\text { Cumbre mundial sobre Ambiente y De- } \\
\text { sarrollo de Johannesburgo (ONU) }\end{array}$ \\
\hline 2005 & $\begin{array}{l}\text { Evaluación de los Ecosistemas del Mile- } \\
\text { nio (ONU) }\end{array}$ \\
\hline 2006 & $\begin{array}{l}\text { Informe Stern sobre impactos econó- } \\
\text { micos del cambio climático (Reino Uni- } \\
\text { do) }\end{array}$ \\
\hline 2007 & $\begin{array}{l}\text { Panel Intergubernamental de Cambio } \\
\text { Climático (ONU) }\end{array}$ \\
\hline
\end{tabular}

Fuente: elaboración propia del autor.

Aunque el término ha sido criticado por su ambigüedad y porque permite una amplia gama de interpretaciones, la Comisión Brundtland ya dio el primer paso, logrando un apoyo político unánime para su escueta definición (Daly y Cobb, 1997; Jacobs, 1991; Sneddon et al, 2006), pero sigue siendo tarea de investigación, definir las formas de ponerlo en práctica.

\section{Hacia una definición del desarrollo sostenible}

El desarrollo sostenible puede ser entendido de manera global como el mantenimiento o el mejoramiento de las "condiciones de calidad" del sistema de interrelaciones sociedad-naturaleza. Para que estas relaciones sean sostenibles, es necesario que se defina, en la práctica, qué es lo que se pretende sostener: ¿el nivel de desarrollo de la sociedad, independiente del impacto ambiental que se genere?, o ¿el mantenimiento de la base ecológica? a través del respeto a las leyes de la naturaleza como condición para mejorar la calidad de vida de la sociedad, y de los mismos ecosistemas. De estas dos visiones surgen sendas teorías sobre desarrollo sostenible, que originan igualmente diferentes políticas públicas para alcanzarlo y medir su progreso (Turner, 1992; Moffatt et al., 2001; Van den Bergh y Hofkes, 1998): la antrópica, lineal o utilitarista (sostenibilidad débil) que defiende la primera concepción y la ecologista que defiende la necesidad de preservar la base ecosistémica del desarrollo (sostenibilidad fuerte).

\section{Sostenibilidad débill o visión utilitarista de la sostenibilidad} Una expresión matemática puede ayudar a entender mejor el concepto de sostenibilidad, tanto débil como fuerte, a pesar del carácter lineal de la expresión (Guhl, 2006):

$$
\mathrm{Kt}=\mathrm{Kn}+\mathrm{Km}+\mathrm{Kh}+\ldots+\mathrm{Kni}
$$

Para que un proceso sea sostenible debe incrementarse o mantenerse el valor de Kt en el período de tiempo considerado. Donde: Kt, Capital Total, es el valor total de los activos, servicios y productos de los cuales dispone una comunidad, ubicada sobre un territorio dado en un tiempo determinado. Kn, Capital Natural, corresponde al valor de los recursos naturales y servicios ambientales, incluyendo los procesos biofísicos y las relaciones entre los componentes de la ecosfera que proveen servicios de soporte para la vida, en ese territorio. Km, Capital 
Manufacturado, el cual es el valor del capital creado por la sociedad en su actividad. Kh, Capital Humano, entendido como la capacidad cognoscitiva de la sociedad para contribuir a su desarrollo incluyendo el conjunto de normas, reglas y costumbres que hacen posible que la comunidad viva y progrese de manera armoniosa y pacífica. Kni, otras formas de capital.

El planteamiento de la sostenibilidad débil considera que el agotamiento del Capital Natural (Kn), se compensaría con una acumulación suficiente de Capital Manufacturado (Km) [Solow, 1974; Stiglitz, 1974 y Hartwick, 1977]. El supuesto que yace de por medio es que entre ambas formas de capital existe una perfecta sustitución y que para mantener niveles de producción y de consumo crecientes en el tiempo, el agotamiento del $K n$ puede ser compensado con un aumento suficiente de $K m$. Entonces, para mantener o incrementar el stock de Capital Total (Kt), lo fundamental no sería conservar el $K n$, sino aumentar el $\mathrm{Km}$ para que no decayese el consumo, principal objetivo de la sostenibilidad débil. Así, si se tala un área selvática y se desarrolla un proyecto agrícola que genere un valor monetario por lo menos igual al dado al bosque, probablemente tasado solamente como madera, los defensores de la "sostenibilidad débil" dirán que esta actividad es sostenible (Guhl, 2006).

Bajo esta visión, el progreso técnico junto al cambio en los precios relativos, pueden hacer que el $\mathrm{Km}$ reemplace cualquier tipo de $K n$, por lo cual no existirían restricciones ambientales al crecimiento económico, llamándose por ello un modelo de "optimismo económico/ tecnológico" (Van Kooten y Bulte, 2000). Esta perspectiva es catalogada como débil, por que no da un tratamiento especial al capital natural, evadiendo el problema de su agotamiento, al suponer que otras formas de capital y el progreso tecnológico siempre podrán compensarlo (Cabeza, 1996; Victor, 1991; Martínez-Alier y Roca, 2001). Como puede observarse, esta concepción se dirige a sostener los fines antrópicos y utilitaristas del desarrollo tales como consumo, bienestar o ingreso per cápita, los cuales no deberían decrecer en el tiempo. Con esta visión, se pasa de una posición determinante de la dimensión ecológica en el concepto de sostenibilidad, a otra subordinada, negando que la economía esté condicionada por los límites naturales al quedar separadas ambas dimensiones, manteniendo el estatus tradicional de la ciencia económica como ciencia autónoma, ajena a las demás ciencias (Bermejo, 2005).

Esta visión resulta ser la más generalizada e institucionalizada encontrando respaldo en los diferentes organismos internacionales como el Banco Mundial, el FMI, la OECD, y la mayor parte de los gobiernos de los diferentes países del mundo. Dentro de su estructura analítica y su cosmovisión en la relación sociedad-naturaleza, la sostenibilidad débil identifica a los tres sistemas básicos de todo proceso de desarrollo, el natural, el económico y el social, como sistemas autónomos, los cuales pueden ser tratados independientemente (reduccionismo), desconociendo los límites relacionados con la capacidad del sistema ambiental (ver figura 1). Además plantea que las zonas donde los tres sistemas interactúan son el área de integración donde la sostenibilidad es alcanzada, mientras que el área por fuera de esta zona es asumida como un área de contradicción y conflicto (bivalente). 
Figura 1. Cosmovisión de la sostenibilidad débil

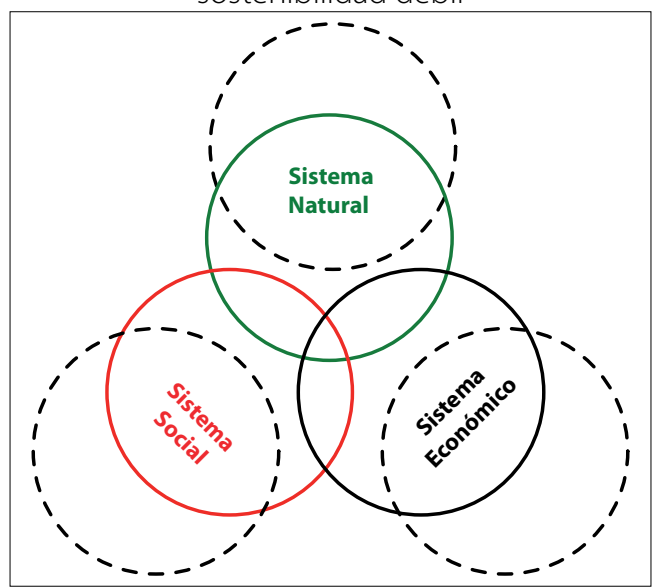

Fuente: falta información

La plataforma de solución para este planteamiento está relacionada con el crecimiento económico que proveerá los recursos necesarios para disminuir la pobreza, financiar soluciones tecnológicas guiadas por el principio de la eco-eficiencia y mejorar el financiamiento del estado y la capacidad de las instituciones vinculadas al sector ambiental (curva ambiental de Kuznets). Aunque en algunos casos estas recomendaciones pueden ser necesarias, en muchos otros resultan contraproducentes, dada la existencia de límites naturales y el carácter complejo e irreversible de muchas de las interrelaciones entre el mundo natural y el mundo social. De tal manera, no se considera la "sostenibilidad débil" como un marco conceptual adecuado para el diseño de políticas públicas para el desarrollo sostenible en Colombia.

De la misma manera, surgen metodologías para definir el nivel de sostenibilidad de los países. Desde esta perspectiva, es factible reemplazar el capital natural por el capital creado por la sociedad; y además es posible valorar todas las externalidades ambientales en términos de depreciación del capital natural, porque un país es más sostenible si la tasa de ahorro es superior a la depreciación de este tipo de capital más la depreciación del capital creado por la sociedad. Bajo este enfoque, los países más ricos y con menor abundancia de recursos naturales, resultan los más sostenibles y los países más pobres y regularmente con mejor dotación ambiental, son los más insostenibles ambientalmente. Este argumento igualmente supone que no hay transferencia de costos ambientales entre unos países y otros a través del comercio internacional.

\section{Sostenibilidad fuerte o sostenibilidad de la base ecológica}

\section{Aspectos generalles}

Los ecosistemas naturales, incluidos los sistemas dominados por los humanos, corresponden a "sistemas complejos adaptados". Debido a que estos sistemas son evolutivos antes que mecánicos, tienen un grado limitado de previsibilidad. Los sistemas ecológicos juegan un papel fundamental a la hora de apoyar la vida en la tierra sin la cual la actividad económica no sería posible. A la larga, una sociedad saludable sólo puede existir y sostenerse en simbiosis solamente con una ecología saludable. Estas dos dimensiones son tan interdependientes que, aislarlas para un propósito académico o utilitarista, ha llevado a la tergiversación y a una pobre gestión ambiental (Costanza et al., 1999).

En tal sentido, la sostenibilidad fuerte parte del hecho de considerar la imposibilidad de sustitución de muchas de las funciones y servicios ambientales. El resultado de estas consideraciones es que 
el $K n$ y el $K m$ deben mirarse como complementarios y no como sustituibles. Las presunciones de la casi perfecta sustitución entre $K n$ y $K m$ son una grave distorsión de la realidad, donde la excusa de la "conveniencia analítica" no la justifi$\mathrm{ca}$, pues, como señala Georgescu-Roegen (1980), más allá de los factores productivos estarán siempre los recursos naturales y los servicios ambientales.

Bajo este modelo, lo que se debe sostener son los medios para mantener o conservar la capacidad del ambiente para soportar los patrones de desarrollo que se quieren alcanzar; es decir, la base ecosistémica que soporta el desarrollo socioeconómico. Para alcanzar esto, es necesario considerar la existencia de unos límites y unas leyes naturales que es necesario respetar. Los límites que la visión de la sostenibilidad fuerte impone al aprovechamiento de los recursos naturales y servicios ambientales, están definidos por la capacidad de la naturaleza para reproducirse y para autopurificarse y de esta manera, absorber o eliminar la contaminación. Así, si un bosque se explota a una tasa de extracción mayor a su capacidad de regeneración, se estará consumiendo el capital natural más allá de lo que pudiéramos llamar los "rendimientos" de este capital, lo que implica un desarrollo insostenible (Guhl, 2006).

La sostenibilidad fuerte se sustenta en el "escepticismo tecnológico" y en el "principio de precaución". La tecnología no será capaz de evitar las restricciones fundamentales de energía y recursos, y finalmente el crecimiento económico material deberá cesar (Daly, 1989). La gran incertidumbre sobre los impactos de las restricciones de energía y recursos y el enorme tamaño de lo que está en juego (la sobrevivencia de la humanidad), hace que debamos ser precavidos a la hora de creer que la capacidad tecnológica hará desaparecer las restricciones de recursos ${ }^{4}$.

La cosmovisión en la que se sustenta la sostenibilidad fuerte, es la mirada ecosistémica de los componentes con base en un modelo de interdependencia cósmica a partir de la Teoría General de Sistemas (Mebratu, 1998). Este enfoque nos dice que el universo en general, y el cosmos económico y social en particular, nunca han sido ni serán, sistemas separados del sistema natural, pues es necesario concebirlos integradamente (figura 2). El área de intersección de los diferentes cosmos que la integran, es el área donde hay millones de combinaciones de conflictos y armonías sirviendo como un semillero para los procesos de coevolución del universo natural y humano. Bajo esta visión, las funciones y servicios ambientales corresponden al soporte o la base donde opera o se asienta la sociedad y la actividad económica. Por esta razón, es la base ambiental y ecológica lo que se requiere sostener para posibilitar que el desarrollo

4 Sobre este tema, una aportación interesante desde la sociología ambiental es el concepto de "sociedad del riesgo" de Beck, U. (1992). La hipótesis central de este argumento es que el desarrollo de la sociedad moderna la ha llevado a desembocar en una fase en que los riesgos sociales, políticos, económicos e individuales tienden a ponerse cada vez más fuera del alcance de las instituciones establecidas para su supervisión y control. El principio organizador de la sociedad industrial fue la distribución de los bienes; el de la "sociedad del riesgo" es la distribución, prevención, control y legitimación de los "males", esto es de los peligros que acompañan las nuevas tecnologías, el deterioro del medio ambiente, la supermilitarización y la pobreza de la periferia (Beck, 1992). 
socio-económico se pueda mantener en el tiempo.

Para la sostenibilidad fuerte, el crecimiento económico debe ser reducido y hacerse compatible con la dinámica de crecimiento de los recursos naturales renovables y el desarrollo de recursos y fuentes energéticas alternativas que posibiliten la sostenibilidad de los recursos no renovables. Si la escala de la economía es muy grande y su velocidad es muy rápida, ésta puede superar la capacidad de asimilación y regeneración de los ciclos naturales afectando la sostenibilidad del desarrollo. En tal sentido, como lo afirma Bermejo (2005):

"al ser la especie humana parte de la naturaleza, la economía humana no puede ser más que un subsistema de la economía general de los materiales y la energía, que es el componente no vivo de la ecología. El principio de jerarquía natural determina que el subsistema no puede transgredir las normas del sistema, así que solo será sostenible cuando imite a la Naturaleza. Esto supone convertir los comportamientos naturales en principios guías de nuestra economía y sociedad”.

La principal escuela teórica que respalda la sostenibilidad fuerte es la Economía Ecológica (EE), la cual es un nuevo campo transdiciplinario que mira a la economía y a la sociedad como un subsistema de un sistema mucho más grande, finito y global que es la biosfera. Este enfoque, basado en el análisis ecosistémico, nos dice que el universo en general, y el cosmos económico y social en particular, nunca han sido ni serán, sistemas separados del sistema natural, por lo que hay que concebirlos integradamente. En este sentido, los subsistemas económico y social están abiertos tanto a la entrada y salida de energía y materiales como a la salida de desperdicios y emisiones desde y hacia la biosfera. La disponibilidad de energía libre y el ciclo de materiales posibilitan formas de vida cada vez más organizadas y complejas, lógica que aplica perfectamente a la economía y la sociedad. Energía disipada y desperdicios son producidos en el proceso. Una parte menor de los desperdicios pueden ser reciclados y cuando no, la sociedad y la economía toman nuevos recursos. Pero también, la EE ve a la economía y a la sociedad no solo empotrada en el ecosistema, sino en la histórica y cambiante percepción social de los ecosistemas. Además, está empotrada en una estructura de derechos de propiedad sobre los recursos y servicios ambientales, en una distribución social del poder y de los ingresos, en una estructura de género, social, de clases y de castas (Martínez-Alier, 2002).

Figura 2. Cosmovisión de la sostenibilidad fuerte

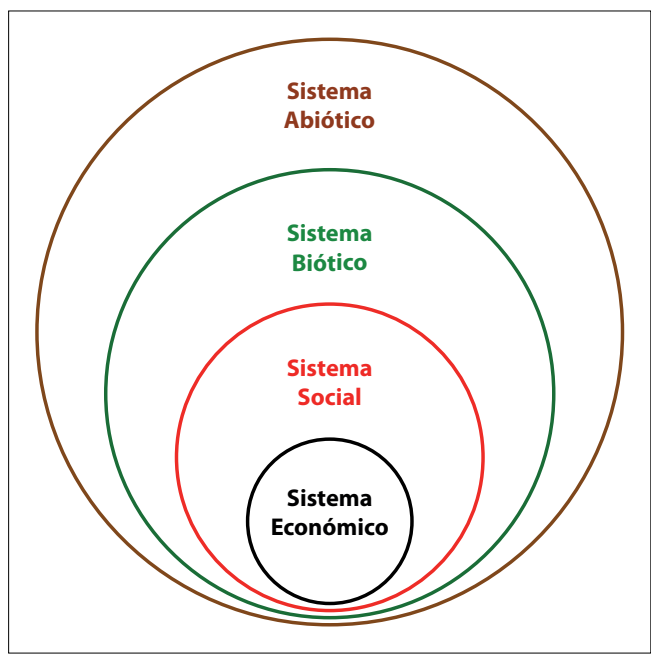

La EE es también la ciencia que estudia las relaciones y conflictos entre los sistemas económicos y los ecosistemas, 
partiendo de creer en los límites ambientales y estudiando también la coevolución de las especies con los seres humanos. El objeto básico de estudio de la EE es la (in)sostenibilidad ecológica de la economía, sin recurrir a un solo tipo de valor expresado en un único numerario, incluyendo así, la evaluación biofísica de los impactos ambientales de la economía humana.

Precisamente esto permite que se destaque el uso de indicadores que permitan identificar el grado de agotamiento y uso de los RN, dado que en el fondo, la sostenibilidad dependerá del tamaño que la economía ocupe dentro del conjunto de la biosfera; y una buena forma de medir ese tamaño o "escala" en términos físicos, resulta de contabilizar los flujos de energía y materiales que recorren la economía de un país, permitiendo así conocer las bases materiales en la cual se sustenta la sociedad. Bajo esta realidad, aparecen indicadores como la Huella Ecológica (Rees \& Wackernagel, 1996), la Huella Hídrica (Chapagain \& Hoekstra, 2004), el Análisis de Flujo de Materiales (EUROSTAT, 2001), el Balance Energético (Giampietro, 1999), entre otros.

Siendo así, tanto la gestión ambiental como las políticas de las autoridades ambientales, deberán tener como objetivo el mantenimiento en el tiempo de la capacidad de la biosfera con el fin de que esta pueda continuar desarrollando las tres funciones ambientales esenciales: a) como abastecedora de recursos naturales, b) como asimiladora de desperdicios y contaminación, y c) como prestadora de servicios ecosistémicos para la vida.
Interrelaciones ambientales entre territorios: mirada global y regional de la sostenibilidad fuerte

\section{Visión global de la sostenibilidad}

Debido a que los subsistemas social y económico dependen de las funciones ambientales y estas últimas están perfectamente interrelacionadas, es necesario ver la biosfera como una sola unidad planetaria. Ello obliga a que la discusión sobre la sostenibilidad deba adoptar una perspectiva global de carácter dinámico, en el cual la dinámica de las economías y sociedades locales (nacionales) y sus patrones de consumo se transfieren o se cargan ambientalmente a otras realidades locales a través del comercio ya sea nacional o internacional. El comercio entre naciones y regiones ofrece una forma de salvar la restricción ecológica importando servicios y recursos ambientales (incluyendo la absorción de desechos) de otras partes. Esto lleva a la situación en donde cada país o región trata de vivir por encima de su propia capacidad de absorción y regeneración importando estas capacidades de otros sitios, haciendo que los problemas ambientales de tipo local se nacionalicen $o$ internacionalicen y viceversa (Costanza et al., 1999). Así, el comercio (entre regiones o internacional) juega un rol importante en la intensificación y distribución de las cargas e impactos ambientales entre países (y regiones), porque puede ser considerado como el nuevo vector (igual al aire y al agua) que disemina las cargas e impactos ambientales desconociendo las fronteras (Karlson, 1995).

De esta forma, la discusión sobre sostenibilidad se conecta claramente con la preocupación por la equidad ambiental entre regiones del mundo y al interior de los países, dando lugar al concepto de deuda 
ecológica, entendida ésta como el espacio ambiental de un país (o región) que es usufructuado por otro país (o región) (v.gr. el calentamiento global) [Pérez, 2007].

\section{Visión regional de la sostenibilidad}

Por otra parte, es importante reconocer que el análisis de la sostenibilidad no puede ser entendido de una manera simplista: un territorio específico equivales a un país, como una unidad monolítica nacional homogénea, puesto que el concepto de Nación corresponde, en la práctica, a una división histórica de carácter político-administrativo, que además tiene un alto grado de heterogeneidad socio-cultural entre regiones. Precisamente, el análisis de la realidad ambiental requiere para profundizar en su conocimiento el uso del concepto de región ambiental que permite establecer las relaciones que en un espacio determinado dentro de un país se han desarrollado entre la naturaleza y la sociedad que la habita y usufructúa. Para facilitar estos análisis, las ciencias ambientales han construido el concepto de ecoregión y dentro de él los ecosistemas estratégicos. La ecoregión y sus ecosistemas estratégicos, deben convertirse en la unidad biofísica-cultural de análisis de la problemática ambiental de un país, y en el eje de la planificación ambiental y de su política pública.

\section{Operacionalización conceptual del desarrollo sostenible en Colombia}

Teniendo en cuenta las consideraciones anteriores, planificar el desarrollo sostenible implica identificar las formas más adecuadas de utilizar los recursos naturales y servicios ambientales, de manera que se garantice su continua provisión para las actividades productivas y la estabilidad de los ecosistemas para que no ocurran fenómenos irreversibles, y la permanencia de unas condiciones ambientales en niveles tolerables, para proteger la salud humana. Cada país debe estudiar la forma de adecuar estas teorías y herramientas a la solución de su problemática particular y trabajar en la definición de criterios para evaluar la sostenibilidad, la cual es entendida como la permanencia de unas soluciones en el tiempo que requiere de un tratamiento dinámico y sistémico de la información disponible.

El enfoque de dinámica de sistemas resulta una herramienta poderosa para encarar no solo la complejidad que presenta el problema del desarrollo sostenible, sino que también es un instrumento útil para diseñar la política pública en este campo, teniendo en cuenta tanto el papel de Colombia como el país periférico en los arreglos políticos y económicos a nivel mundial, como en la necesidad de establecer una política ambiental por ecoregiones. Acorde a la segunda ley de la teoría de sistemas, un sistema se organiza en niveles jerárquicos, ordenados de mayor a menor: biosfera, región biogeográfica, bioma, paisaje, ecosistema, comunidad biótica, población, organismo, órgano, célula (Bermejo, 2001). Cada nivel de jerarquía ejerce su influencia en los niveles inmediatamente adyacentes de forma que los procesos en los niveles inferiores están influidos por los que se dan en los superiores (Odum, 1992). Cada subsistema natural está sometido a la jerarquía del sistema del que forma parte y somete a las partes que lo forman. Sin embargo, esta "subordinación entre niveles es 
siempre incompleta y cada nivel puede tener sus propias normas de comportamiento y sus propias relaciones" (Gowdy, 1999). Esto da a los subsistemas un grado de autonomía que es necesario construir, ejercer y defender.

Con base en lo anterior, la planificación, gestión y ejecución de la política pública para el desarrollo sostenible en Colombia, debe basarse en un análisis ecosistémico desde el punto de vista de la sostenibilidad fuerte que permita entender tanto las relaciones de dependencia del país como la existencia de ecoregiones donde existen problemas ambientales específicos que es importante resolver a través de la política pública. Existen en el país cinco ecoregiones claramente delimitadas y analizadas con sus respectivos ecosistemas estratégicos: Andina; Caribe que incluye la insular; Pacífica; Amazonía y Orinoquía. Pueden subdividirse para efectos de planificación, creando unidades espaciales menores alojadas en las grandes regiones naturales. Por ejemplo, el Cinturón Cafetero o el Valle del Cauca en la Región Andina.

Este enfoque permite entender a Colombia como un sistema abierto, tanto en términos ambientales, como socio-económico-culturales y políticos. En términos ambientales, está abierto a la entrada de flujos de energía, materiales, residuos y emisiones desde el sistema natural y desde la actividad económica externa al país y hacía los sistemas natural, social y económico internos. Esta realidad es la que permite trasladar los impactos ambientales desde el nivel global al nivel local (nacional) a través de diferentes mecanismos: el flujo de bienes y servicios del comercio internacional; los problemas ambientales globales que tienen efectos sobre la biosfera nacional, etc. Además está abierto a la salida de materia y energía, residuos y emisiones desde Colombia hacia el resto del mundo. Por su parte, las ecoregiones incluyen sus respectivos subsistemas sociales y económicos, los cuales además están integrados al sistema socio-económico nacional, generando una red de dependencia e interrelaciones entre las ecoregiones, las cuales también están abiertas al sistema económico mundial (SEM).

Desde el punto de vista socio-económico-cultural y politico, el país puede considerarse como una nación dependiente o con bajo nivel de gobernabilidad en cuanto a decisiones en el campo económico, cultural, político, ambiental y social. Pero posee fortalezas ambientales importantes que deben incorporarse a una política de desarrollo sostenible. Es decir, dada la estructura asimétrica de la economía y del poder a nivel mundial, el país depende de muchas decisiones que son tomadas por fuera de su orbita y de su gobernabilidad. Pero igualmente, algunas decisiones a nivel mundial como Convenios o propuestas de Política Internacional, pueden afectar positivamente el desarrollo sostenible de Colombia. Todo ello debe considerarse a la hora de diseñar propuestas de política pública para promover el desarrollo sostenible en el país.

Finalmente, bajo la óptica de la sostenibilidad fuerte, lo que es necesario sostener es la base que soporta o sustenta las actividades sociales y económicas; es decir la base ambiental y ecológica. Por tal razón, las políticas públicas de tipo ambiental deben concebirse en su interrelación con los subsistemas social y económico, y deben orientarse a buscar que la base ecológica se sostenga en el tiempo. Solo respetando las restricciones ambientales y los ritmos y leyes de la Naturaleza, 
el país puede mejorar en forma sostenible sus niveles de desarrollo económico y social, y caminar por una senda segura hacia el desarrollo sostenible. Es necesario señalar además que Colombia posee un importante espacio en su estructura legal y constitucional para promocionar el desarrollo sostenible.

\section{Conclusiones}

Para diseñar una adecuada política pública para el desarrollo sostenible acorde al marco conceptual planteado, es necesario el respeto a una serie de principios guías que buscan hacer efectiva y aplicable la política pública en este campo.

- Un principio general que enmarca la política pública en el campo ambiental tiene que ver con el respeto a las leyes y normas de la naturaleza, aspecto que lleva implícito la existencia de límites y de órdenes jerárquicos donde la sociedad hace parte de la biosfera, la cual tiene una jerarquía superior. Esto supone convertir los procesos naturales en principios guías de nuestra actividad económica y social para alcanzar el desarrollo sostenible (DS). Además, la aplicación del principio de precaución es un elemento esencial de la política pública de DS, dado el carácter irreversible y difícilmente predecible de algunos impactos sobre el capital natural, situación que pone en riesgo las funciones del territorio como soporte de vida.

- El reto del DS exige pasar de los tradicionales modelos de gestión ambiental como simples recursos, a nuevos modelos de gestión ecosistémica, en donde se aprecie la multitud de servicios ambientales que provee la naturaleza para el soporte de la vida; se observen los frágiles equilibrios de las funciones de los ciclos naturales y la compleja pirámide de vida que albergan; se reconozcan los valores sociales, culturales y de identidad, tanto territorial como colectiva; se recupere el sentido lúdico y el valor estético de la naturaleza. Todo ello, sin olvidar la necesidad de gestionar con eficiencia, las utilidades económicas de los mismos como recursos productivos.

- Para alcanzar el DS, es esencial actuar sobre variables estructurales relacionadas con los patrones de desarrollo y de consumo, como también en nuestra relación cultural con el ambiente. Aunque este tipo de políticas (educación ambiental y cambio cultural), tienen efectos más lentos, sus impactos son más duraderos para la sostenibilidad en el largo plazo. De allí que la popularización del conocimiento sobre el tema ambiental que permita informar, educar y difundir en todos los niveles y escalas, la problemática ambiental es una necesidad para promover la sostenibilidad.

- Una condición necesaria para el éxito de la política de DS es su carácter integral y transversal de tal manera que se inserte en las diferentes políticas y estrategias sectoriales y nacionales. Por eso, esta política debe formularse en concertación con los diversos sectores y actores que hacen parte de esos procesos.

- Solo puede avanzarse hacia la sostenibilidad si la sociedad define y aplica planes de largo plazo para el DS. Además, la probabilidad de éxito de las políticas de DS se incrementa, a través 
de la implementación de estrategias y acciones descentralizadas. Por esto, la visión de ecoregión debe involucrarse en la formulación y aplicación de políticas ambientales, teniendo en cuenta la heterogeneidad natural, cultural y social de dichas áreas.

- Una de las causas explicativas de la problemática ambiental está relacionada con la falta de conocimiento sobre el mundo natural y sus capacidades. Por ello, el desarrollo de una sólida agenda de conocimiento e investigación es una estrategia fundamental para el DS.

- La ecotecnología contribuye de manera importante a disminuir los impactos ambientales resultados de las actividades económicas, pero no es una condición suficiente para alcanzar el DS puesto que el cambio tecnológico no es un perfecto sustituto del capital natural. Acá, es igualmente útil desincentivar el uso de patrones tecnológicos recurso-intensivos.

- Dada nuestra situación de dependencia internacional, es necesario promover políticas autónomas y soberanas en el campo económico, cultural y político, que sean conscientes, informadas y responsables de sus implicaciones ambientales y orientadas a limitar el traslado de los costos ecológicos a nuestro territorio.

- El conocimiento y la solución de los problemas ambientales, considerando el análisis del entorno, es una estrategia adecuada para promover la sostenibilidad, puesto que sin un análisis global, lo que se consigue es un trasvase de la carga ambiental, entre instalaciones, regiones, países, sectores económicos, vectores ambientales, categorías de impacto ambiental o en el tiempo, y no su reducción.

- La política pública orientada al DS en Colombia enfrenta el reto de armonizar la búsqueda de una mejor calidad de vida de la población con la necesidad de conservar la extensa y rica base ecosistémica del país. Esto hace de la política ambiental una tarea compleja que obliga, a que la misma, deba construirse dentro de una estructura de red, que refleje su interactividad y carácter sistémico, promoviendo la integración y coordinación entre las políticas misionales y las instrumentales.

\section{Bibliografía}

1. Barán, P. y Hosbawn, J. (1958). Las etapas del crecimiento económico. El Trimestre Económico, n. 97, pp.63-71. México: F.C.E.

2. Beck, U. (1992). Risk society: towards a new modernity. London: Sage.

3. Bermejo, R. (2001). Economía sostenible: principios, conceptos e instrumentos. Bilbao: Bakeaz.

4. Bermejo, R. (2005). La gran transición hacia la sostenibilidad: principios y estrategias de economía sostenible. Madrid: Catarata.

5. Bertalanffy, L. (1976). Teoría General de Sistemas. Fundamentos, desarrollos y aplicaciones. México: FCE. (La versión original en inglés data de 1968).

6. Boada, M. y Toledo, V. (2003). El planeta, nuestro cuerpo. La ecología, el ambientalismo y la crisis de la modernidad. México: SEP y FCE.

7. Boulding, K. (1966). The Economics of the Coming Spaceship Earth. University of Colorado at Boulder Libraries. 
8. Cabeza, M. (1996). The concept of weak sustainability. Ecological Economics, 17: 147-156.

9. Cardoso, F. y Faletto, E. (1969). Dependencia y Desarrollo en América Latina. México: Siglo XXI.

10. Carson, R. (1962). Silent Spring. Boston: Houghton Mifflin Company.

11. Chapagain, A. K. y Hoekstra, A.Y. (November 2004). Water Footprints of Nations. Volume 1: Main Report. Value of Water. Research Report Series, n. ${ }^{\circ} 16$. UNESCO-IHE, Delft, The Netherlands. http://www.waterfootprint. org/Reports/Report16.pdf.

12. Costanza, R., Cumberland, J., Daly, H., Goodland, R. y Norgaard, R. (1999). Introducción a la Economía Ecológica. Madrid: AENOR Editorial.

13. Daly, H. (compilador), (1989). Economía, Ecología y Etica: ensayos hacia una economía en estado estacionario. México: FCE.

14. Daly, H. y Cobb, J. (1997). Para el bien común: reorientando la economía hacia la comunidad, el ambiente y el futuro sostenible. México: FCE.

15. Domar, H. (1947). Expansión y empleo. American Economic Review, falta el volumen, el número, pp. 34-55. Ciudad.

16. Ehrlich, P. (1968). The population Bomb. Ballantine: Editorial.

17. Escobar, A. (1994). El desarrollo sostenible: diálogo de discursos. En: Revista Foro, volume, número, pp.98-112.

18. EUROSTAT. (2001). Economy-wide material flow accounts and derived indicators. A methodological guide. Luxemburg: Statistical Office of the European Union.

19. García, E. (2004). Medio ambiente y sociedad. La civilización industrial y los límites del planeta. Madrid: Alianza Editorial.
20. Georgescu-Roegen, N. (1980). "Selecciones de "mitos de la economía y de la energía”. Daly, H. (comp.). En: Economía, Ecología y Ética. Ensayos hacia una economía e estado estacionario. México: FCE.

21. Giampietro, M. (1999). Energy use in agriculture. Encyclopedia of life sciences. MacMillan Publisher.

22. Gowdy, J. (1999). Hierarchies in human affairs: Microfoundations of Environmental Sustainability. En: Kohn, J. y otros: Sustainability in Questions. London: Edward Elgar.

23. Guhl, E. (2006). Reflexiones sobre la sostenibilidad. Disponible en www.quinaxi.com.co. Sin publicar.

24. Guhl, E. (en imprenta). La huella bumana y la sostenibilidad. Ciudad: Universidad Nacional.

25. Jacobs, M. (1991). Economía verde: medio ambiente y desarrollo sostenible. Bogotá: TM Editores y Ediciones Uniandes.

26. Hartwick, J. (1977). Intergenerational equity and the investing of rents from exhaustible resources. American Economic Review, 67, pp.972-974.

27. Karlson, R. (Diciembre 1995). Recycling in life cycle assesments. Tesis doctoral. Göteborg: Chalmers University of Thecnology.

28. Martínez-Alier, J. y Roca Jusmet, J. (2001). Economía Ecológica y Politica Ambiental. México: FCE.

29. Martínez-Alier, J. (2002). The environmentalism of the poor. A study of ecological Conflicts and Valuation. Edward Elgar, Cheltenham.

30. Mebratu, D. (1998). Sustainability and sustainable development: historical and conceptual review. Environmental impact assessment review, n. ${ }^{\circ} 18$, pp.493520. 
31. Meadows, D., Meadows, D. y Randers J. (1972). Los limites del crecimiento. Madrid: El País-Aguilar.

32. Moffatt, I., Hanley, N. Wilson, M. (2001). Measuring and modeling sustainable development. Ciudad: The Parthenon Publishing Group Inc.

33. Naredo, J. M. (2003). La economía en evolución: historia y perspectivas de las categorias del pensamiento económico. $3^{\text {a }}$ edición actualizada. Madrid: Siglo XXI Editores.

34. Odum, E. P. (1992). Ecologia: bases cientificas para un nuevo paradigma. Barcelona: Vedrá.

35. Pérez, M. (2007). Comercio internacional y medio ambiente en Colombia: mirada desde la economía ecológica. Cali: Ediciones Universidad del Valle.

36. Pezzy, J. (1989). Economic analysis of sustainable growth and sustainable development. Environmental Department. Working paper n. ${ }^{\circ} 15$. Washington D.C.: The World Bank

37. Prebisch, R. (1949). El desarrollo económico de la América Latina y algunos de sus principales problemas. Santiago de Chile: CEPAL, E/CN.12/89. Publicado en inglés en 1950 bajo el título: "The Economic Development of Latin America and its Principal Problems. New York: UNCLA.

38. Ranis, G. y Fei, J.C. (1952). Una teoría del desarrollo económico. El Trimestre Económico, pp. 246-271. México: F.C.E.

39. Rees, W. \& Wackernagel, M. (1994). Ecological footprints and appropriated carrying capacity: Measuring the natural capital requirements of the human economy. En Jansson, A-M., M. Hammer, C. Folke, and R. Constan$\mathrm{za}$ (Eds.). Investing in natural capital: The ecological economics approach to sustainability, pp.362-390. Washington: Island Press.

40. Rostow, W. (1956). Las cinco etapas del crecimiento. En: Las etapas del crecimiento económico: un manifiesto no comunista, pp. 16-25. México: FCE.

41. Sachs, W. (1996). La anatomía política del desarrollo sostenible. En: La gallina de los huevos de oro: debate sobre el concepto de desarrollo sostenible, pp.15-43. Bogotá: Ecofondo y Cerec.

42. Schumpeter, J. (Mayo 1935). The Analysis of Economic Change. The Review of Economics Statistics, vol. XVII, no 4, pp. 2-10. Falta ciudad.

43. Sneddon, C., Howarth, R. y Norgaard, R. (2006). Sustainable development in a post-Brundtland world. Ecological Economics, n. ${ }^{\circ} 57$, pp.253-268.

44. Solow, R. (1956). Una contribución a la teoría del crecimiento económico. Quautpuly Journal of Economics, pp.6594.

45. Solow, R. (January 1974). Intergenerational Equity and Exhaustible Resources. The review of economic studies, $\mathrm{n}$. 41 faltan páginas. Ciudad.

46. Stiglitz, J. (January 1974). Growth with Exhaustible Natural Resources: Efficient and Optimal Growth Paths. The review of economic studies, n. ${ }^{\circ} 41$, pp.?? Ciudad.

47. Turner, R. (1992). Speculations on weak and strong sustainability. CSERGE Working paper. GEC 92-96, CSERGE. Norwich: University of East Anglia.

48. Van den Bergh, J. y Hofkes, M. (1998). A survey of economic modeling of sustainable development.

49. Van Kooten, G. y Bulte, E. (2000). The economics of nature: managing biological assets. Blackwell Publishers, Oxford. 
50. Victor, P. (1991). Indicators of sustainable development: some lessons from capital theory. Ecological Economics, n. ${ }^{\circ}$, pp.191-213.

51. Wallerstein, I. (1974). The Modern World System: Capitalist Agriculture and the Origins of the European World Economy in the Sixteenth Century, pp. 229-233. New York: Academic Press.

52. WCED. (1987). Our Commun Future, Oxford: Oxford University Press. 